\title{
Cumulative team experience matters more than individual surgeon experience in cardiac surgery
}

\author{
Andrew W. ElBardissi, MD, MPH, MBA, ${ }^{\text {a }}$ Antoine Duclos, MD, PhD, ${ }^{\text {a,d } J a m e s ~ D . ~ R a w n, ~ M D, ~}{ }^{\text {b }}$ \\ Dennis P. Orgill, MD, PhD, ${ }^{\mathrm{a}, \mathrm{c}}$ and Matthew J. Carty, $\mathrm{MD}^{\mathrm{a}, \mathrm{c}}$
}

\begin{abstract}
Objectives: Individual surgeon experience and the cumulative experience of the surgical team have both been implicated as factors that influence surgical efficiency. We sought to quantitatively evaluate the effects of both individual surgeon experience and the cumulative experience of attending surgeon-cardiothoracic fellow collaborations in isolated coronary artery bypass graft $(\mathrm{CABG})$ procedures.
\end{abstract}

\begin{abstract}
Methods: Using a prospectively collected retrospective database, we analyzed all medical records of patients undergoing isolated $\mathrm{CABG}$ procedure at our institution. We used multivariate generalized estimating equation regression models to adjust for patient mix and subsequently evaluated the effect of both attending cardiac surgeon experience (since fellowship graduation) and the number of previous collaborations between attending cardiac surgeons and cardiothoracic fellow pairs on cardiopulmonary bypass and crossclamp times.
\end{abstract}

Results: From 2001 to 2010, 4068 consecutive patients underwent isolated CABG procedure at our institution performed by 11 attending cardiac surgeons and 73 cardiothoracic fellows. Mean attending experience after fellowship graduation was $10.9 \pm 8.0$ years and mean number of cases between unique pairs of attending cardiac surgeons and cardiothoracic fellows was $10.0 \pm 10.0$ cases. After patient risk adjustment, both attending surgical experience since fellowship graduation and the number of previous collaborations between attending surgeons and cardiothoracic fellows were significantly associated with a reduction in cardiopulmonary bypass and crossclamp times $(P<.001)$. The influence of attending-fellow pair experience far exceeded the influence of surgical experience with beta estimates for attending-fellow pair experience nearly three times that of attending surgeon experience.

Conclusions: Cumulative experience of attending cardiac surgeons and cardiothoracic fellows has a dramatic effect on both cardiopulmonary bypass and crossclamp times, whereas attending cardiac surgeon learning curves following fellowship graduation are clinically insignificant. Taken together, these findings suggest that the primary driver of operative efficiency in CABG procedure is the collaborative experience of the attending surgeon-cardiothoracic fellow operative team, rather than the individual experience of the attending surgeon.

(J Thorac Cardiovasc Surg 2013;145:328-33)

The application of quality improvement principles to the discipline of surgery has yielded a wide body of work that has provided insight into the influence of team dynamics on surgical performance. Previous studies have suggested that quantitative and behavioral methods ${ }^{1}$ can be applied to the surgical arena to qualify the effectiveness of surgical performance, thereby allowing for the introduction of

From the Center for Surgery and Public Health, ${ }^{\mathrm{a}}$ Division of Cardiac Surgery, ${ }^{\mathrm{b}}$ and Division of Plastic Surgery, ${ }^{c}$ Brigham \& Women's Hospital, Boston, Mass; and Hospices Civils de Lyon, ${ }^{\mathrm{d}}$ Pôle Information Médicale Evaluation Recherche-Université de Lyon, EA Santé-Individu-Société 4129, Lyon, France.

This work was partially supported by the Agency for Health Care Research and Quality National Research Service Award grant No. 1F32HS019190 and the Arthur and Tracy Cabot Fellowship at Brigham \& Women's Hospital and the Center for Surgery and Public Health. A.W.ElB. receives salary support from this contract.

Disclosures: Authors have nothing to disclose with regard to commercial support.

Received for publication June 17, 2012; revisions received Sept 2, 2012; accepted for publication Sept 13, 2012; available ahead of print Oct 19, 2012.

Address for reprints: Andrew W. ElBardissi, MD, MPH, MBA, 75 Francis St, Boston, MA 02115 (E-mail: aelbardissi@post.harvard.edu).

$0022-5223 / \$ 36.00$

Copyright (c) 2013 by The American Association for Thoracic Surgery

http://dx.doi.org/10.1016/j.jtcvs.2012.09.022 focused strategies aimed at improving surgical performance and ultimately patient safety and outcomes. A recent application of a human factors methodology to the cardiac surgery operating room found that teamwork-related failures were strongly correlated with surgical errors. ${ }^{2}$ A subsequent analysis found that these teamwork failures were closely related to the composition of the team; team members with prior experience working together had far fewer teamwork-related disruptions and consequently fewer surgical errors compared with teams of people who were unfamiliar with each other. ${ }^{3}$ These reductions in error are likely the result of the team's cumulative experience. ${ }^{4}$

Although many studies emphasize the importance of highly effective team dynamics (which can be further accentuated by a team's cumulative experience), the importance of individual technical proficiency should not be understated. Learning curve analyses have found that surgical performance significantly improves over the course of an individual surgeon's career. ${ }^{5}$ As such, there exists a large body of literature that provides alternate explanations for the improvement in quality and performance that occurs 


\section{Abbreviations and Acronyms \\ $\mathrm{CABG}=$ coronary artery bypass graft \\ $\mathrm{CPB}=$ cardiopulmonary bypass \\ $\mathrm{X}$-clamp $=$ crossclamp}

over the course of a surgeon's career. Furthermore, there are no studies to our knowledge that attempt to reconcile the magnitude of team experience versus individual surgical experience. These data would be extremely useful in a practical sense; should individual technical proficiency far exceed the influence of a team's cumulative experience, approaches to enhance an individual's technical proficiency (ie, coaching and simulation) would allow for an effective intervention to improve surgical performance and quality. Conversely, if cumulative team experience should prove to be the primary culprit in the optimization of surgical performance, management approaches such as effective staffing may provide optimal solutions to enhancing surgical quality.

To study these questions, we attempted to construct a study that would combine traditional approaches utilizing individual surgeon learning curve analyses ${ }^{5}$ with a metric that would capture a team's cumulative experience. The difficulty in accurately capturing the latter metric is quite substantial. Few retrospective clinical and/or administrative databases provide enough granularity to quantify an entire team's cumulative experience. Although prospective approaches to capturing these data remain a viable option, the duration of such a study would be exceptionally long given the need to also capture an individual surgeon's learning curve, which can span 10 years or more. ${ }^{5}$

To address these issues, we utilized a prospectively collected database that captures individual surgeon experience as well as that of the first assistant cardiothoracic fellow. By examining only isolated coronary artery bypass graft $(\mathrm{CABG})$ procedures, we aimed to identify the influence of the attending surgeon's experience as well as the cumulative experience of the unique attending surgeon-cardiothoracic fellow combination on operative efficiency. Although this approach fails to account for the entire team (eg, anesthesiologist, surgical technician, and circulating nurse), it allows for significant insight into the influence of surgical team experience as well as its relationship to an individual surgeon's experience.

\section{MATERIALS AND METHODS}

\section{Study Population and Data Source}

The primary objective of this study was to assess the effect of both the attending surgeon-cardiothoracic fellow pair's cumulative experience and the individual surgeon's experience on surgical efficiency (captured by cardiopulmonary bypass [CPB] time and crossclamp [X-clamp] time) in isolated CABG. Inclusion criteria included all isolated CABG procedures at the Brigham and Women's Hospital performed between December 28,
2001, and December 30, 2010. Exclusion criteria included CABG procedures that were accompanied by concomitant procedures (ie, valve surgery and maze procedure) and any individual records that contained missing data

Preoperative, hemodynamic, operative, and postoperative characteristics were captured via a prospectively collected database modeled after the Society of Thoracic Surgeons ${ }^{6}$ national database criteria. The primary dependent variables included CPB time and X-clamp time. To adjust for patient risk, multivariate regression models, including preoperative and operative variables, were constructed. In addition, attending surgeon experience, defined as the number of years since cardiothoracic fellowship graduation to the date of the procedure, was used to adjust for the attending learning curve. Cumulative experience was defined as the number of isolated $\mathrm{CABG}$ procedures a particular surgeon and cardiothoracic fellow had performed together previously. Following attending surgeon and patient risk adjustment, the overall change that occurred in both $\mathrm{CPB}$ and $\mathrm{X}$-clamp times due to the number of times an attending surgeon-cardiothoracic fellow combination occurred was defined as the influence of cumulative experience on the surgical learning curve. The Brigham and Women's Hospital Institutional Review Board approved this study (protocol 2006p000586).

\section{Statistical Analysis}

To create a patient-level summary measure of case severity and identify significant predictors of both $\mathrm{CPB}$ and X-clamp time, we used linear regression modeling. Covariates for predicting $\mathrm{CPB}$ and $\mathrm{X}$-clamp time included patient age, sex, body surface area, ejection fraction, preoperative dialysis, preoperative atrial fibrillation, cardiogenic shock, immunosuppressive treatment, percutaneous intervention $<6$ hours, preoperative intra-aortic balloon pump or inotrope, peripheral vascular disease, unstable angina (no myocardial infarction $<7$ days), left main disease, cerebrovascular disease/cerebrovascular accident, diabetes, number of diseased vessels, myocardial infarction, operative urgency status, and congestive heart failure/New York Heart Association functional class. Covariates were selected on the basis of having a previously documented association with the length of CPB and X-clamp time ${ }^{7}$ and a low rate of missing data. Model selection to create a robust multivariate patient risk adjustment model independently for both CPB and X-clamp time was performed using the approach described by Collett ${ }^{8}$ and is briefly described here. Following univariate analysis for each preoperative and operative predictor, those with a parameter $P$ value $<.15$ were selected for initial entry into the multivariate model. After initial fitting, nonsignificant variables were eliminated using backward selection $(P<.1)$. Nonsignificant univariate predictors were subsequently tested using forward selection $(P=.1)$ and all possible 2-way interactions were tested using forward selection $(P=.1$ for entering). Finally, all nonsignificant main effects (unless a component of an interaction term) and nonsignificant interactions were removed.

Following adjustment of patient risk characteristics, attending surgeon experience and attending surgeon-cardiothoracic fellow pair experience were entered into each model ( $\mathrm{CPB}$ and $\mathrm{X}$-clamp time) as a continuous variable. The expected performance curve of surgical teams over time was generated based on a multivariate generalized estimating equation regression model, taking into consideration the clustering of patients by attending surgeon. The operative time was the outcome of interest, whereas attending experience and attending surgeon-cardiothoracic fellow pair experience was the predictor and patient case mix was considered as covariate in the final model. Because the operative time curve may not necessarily be a linear function of surgeon experience, we also considered models with quadratic or logarithmic terms in surgeon experience and chose the model with the best fit. ${ }^{9}$ Model estimates were obtained using the GENMOD procedures in SAS version 9.2 (SAS Institute Inc, Cary, NC).

According to model estimates, the expected mean operative time with corresponding minimum and maximum values was displayed as a function of the number of prior collaborations between attending surgeon and cardiothoracic fellow. The expected reductions in operative time associated 
with the attending surgeon's experience or the number of prior collaborations within the surgical team were separately plotted. All tests were 2-tailed.

\section{RESULTS}

Our database included 4068 consecutive patients who underwent isolated $\mathrm{CABG}$ procedure by 11 surgeons and 73 fellows during the 9-year study interval. Mean cumulative experience of discrete attending-fellow combinations was $10.0 \pm 10.0$ cases with a minimum of 1 case and maximum of 62 cases; the distribution was right skewed, as shown in Figure 1. The mean individual attending experience was $10.9 \pm 8.0$ years since cardiothoracic fellowship graduation; however, this distribution was abnormally distributed with a majority of cases being performed by surgeons with $<20$ years of experience and a maximum experience of 39.2 years (Figure 2). Patient demographic, hemodynamic, and operative characteristics are shown in Table 1. Importantly, the mean CPB and $\mathrm{X}$-clamp times were $103.8 \pm 38.8$ minutes and $78.6 \pm 28.9$ minutes, respectively.

Using the backward and forward multivariate regression techniques described above, the CPB multivariate model included patient age, sex, presence of diabetes, preoperative congestive heart failure, preoperative cardiogenic shock, and preoperative ejection fraction. Comparatively, the multivariate patient risk adjustment model created for X-clamp time included only patient, age, sex, and presence of diabetes. Although these multivariate models were statistically significant, they offered little predictive power in capturing the variability in the outcome variables; adjusted $R^{2}$ values for CPB and X-clamp multivariate models were $2.1 \%$ and $1.5 \%$, respectively.

After adjustment for patient risk, attending surgeon experience (in years since fellowship graduation) and attending surgeon-cardiothoracic fellow experience (number of prior cases performed together consecutively) were entered into the multivariate model. The final models are shown in Tables 2 and 3 and Figure 3. In addition, individual fellow experience (in number of cases) was entered into both models to adjust for fellow experience as a determining factor; however, this was highly statistically insignificant $(P>.05)$ and thus removed.

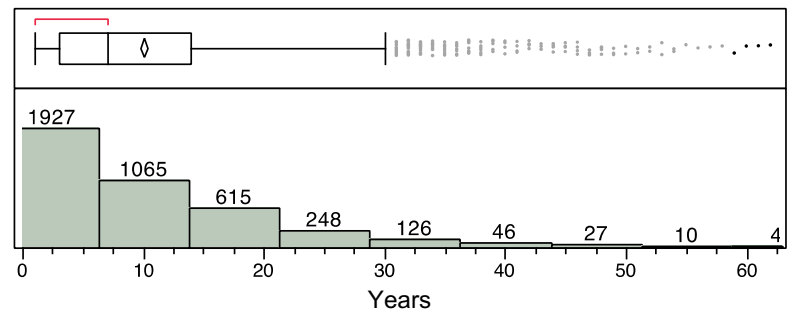

FIGURE 1. Distribution of attending cardiac surgeon-cardiothoracic fellow cumulative experiences.

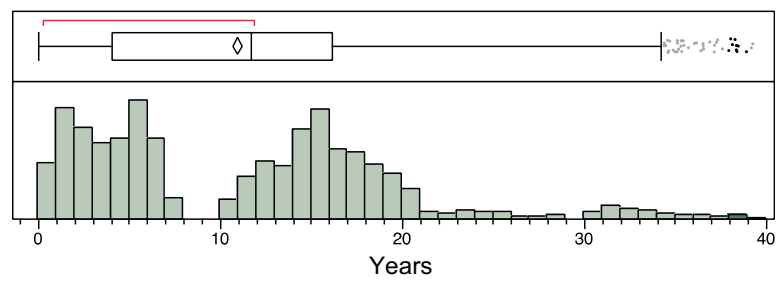

FIGURE 2. Distribution of attending cardiac surgeon experience.

Both attending surgeon experience since fellowship graduation and cumulative attending surgeon-cardiothoracic fellow pair experience were significantly associated with the dependent variables. Addition of these variables offered much greater explanatory variability in predicting the outcome variables, raising the adjusted $R^{2}$ values to $7.2 \%$ for CPB time and $6.1 \%$ for X-clamp time. The influence of attending-fellow pair experience far exceeded the influence of surgeon's experience (Figure 3). The beta estimates for

TABLE 1. Demographic, hemodynamic, and operative characteristics

\begin{tabular}{|c|c|}
\hline Characteristics & Mean or $\%$ \\
\hline \multicolumn{2}{|l|}{ Demographic } \\
\hline Age (y) & $66.3 \pm 10.5$ \\
\hline Male sex & $76(\mathrm{n}=3107)$ \\
\hline Weight (kg) & $85.9 \pm 18.4$ \\
\hline Smoking history & $58.5(\mathrm{n}=2379)$ \\
\hline Diabetes & $37.9(\mathrm{n}=1542)$ \\
\hline Renal failure (preoperative dialysis) & $6.9(\mathrm{n}=282)$ \\
\hline Chronic obstructive pulmonary disease & $10(n=414)$ \\
\hline Cerebrovascular disease & $12.2(\mathrm{n}=497)$ \\
\hline Previous cerebrovascular accident & $5.1(\mathrm{n}=209)$ \\
\hline Congestive heart failure & $24.9(\mathrm{n}=1016)$ \\
\hline Preoperative arrhythmia & $5.2(\mathrm{n}=211)$ \\
\hline \multicolumn{2}{|l|}{ New York Heart Association functional class } \\
\hline I & $19(\mathrm{n}=773)$ \\
\hline II & $44.2(\mathrm{n}=1800)$ \\
\hline III & $28.5(\mathrm{n}=1161)$ \\
\hline IV & $8.2(\mathrm{n}=334)$ \\
\hline Previous coronary artery bypass graft & $13.5(\mathrm{n}=131)$ \\
\hline Previous valve surgery & $1.6 \%(n=16)$ \\
\hline \multicolumn{2}{|l|}{ Hemodynamic } \\
\hline Ejection fraction $(\%)$ & $51 \pm 13$ \\
\hline Pulmonary artery pressure $(\%)$ & $27 \pm 11$ \\
\hline \multicolumn{2}{|l|}{ Operative } \\
\hline \multicolumn{2}{|l|}{ Status } \\
\hline Elective & $45(\mathrm{n}=1829)$ \\
\hline Urgent & $50.8(n=2068)$ \\
\hline Emergent & $4.2(n=169)$ \\
\hline Emergent salvage (\%) & $<1(\mathrm{n}=2)$ \\
\hline Cardiopulmonary bypass time (min) & $103.8 \pm 38.8$ \\
\hline Crossclamp time (min) & $78.6 \pm 28.9$ \\
\hline Internal mammary artery use (any or both) & $94.7(\mathrm{n}=3841)$ \\
\hline Left only & $89.5(n=3642)$ \\
\hline Right only & $0.4(\mathrm{n}=17)$ \\
\hline Both & $4.5(\mathrm{n}=182)$ \\
\hline
\end{tabular}


TABLE 2. Multivariate cardiopulmonary bypass models

\begin{tabular}{lccccc}
\hline & \multicolumn{2}{c}{$\begin{array}{c}\text { Risk-adjustment } \\
\text { model }\end{array}$} & & \multicolumn{2}{c}{$\begin{array}{c}\text { Final cumulative } \\
\text { experience model }\end{array}$} \\
\cline { 2 - 3 } \cline { 6 - 7 } \multicolumn{1}{c}{ Term } & $\begin{array}{c}\text { Beta } \\
\text { estimate }\end{array}$ & Prob $>|\mathbf{t}|$ & & $\begin{array}{c}\text { Beta } \\
\text { estimate }\end{array}$ & Prob $>|\mathbf{t}|$ \\
\hline Intercept & 185.31 & $<.0001$ & & 190.16 & $<.0001$ \\
Age & -0.23 & .0004 & & -0.22 & .0005 \\
Sex & -6.96 & $<.0001$ & -6.99 & $<.0001$ \\
Congestive heart failure & -3.20 & .0489 & -2.98 & .0602 \\
Cardiogenic shock & -19.88 & $<.0001$ & -16.02 & .001 \\
Ejection fraction & -0.13 & .0128 & -0.14 & .0072 \\
Diabetes & -3.32 & .017 & -3.79 & .0052 \\
Attending surgeon & & & -0.33 & $<.0001$ \\
$\quad$ experience & & & & & \\
Attending-fellow team & & & -0.85 & $<.0001$ \\
$\quad$ cumulative experience & & & & \\
\hline
\end{tabular}

attending-fellow pair experience were nearly 3 times that of attending surgeon experience. In other words, for each additional case that an attending-fellow pair performed together, one would expect a $300 \%$ greater reduction in CPB and X-clamp time compared with 1 additional year of attending surgeon's experience. This influence of attending surgeon-cardiothoracic fellow pair experience is further demonstrated by removal of attending surgeon experience from both models; adjusted $R^{2}$ decreased marginally from $7.2 \%$ to $6.9 \%$ for CPB and increased from $6.1 \%$ to $6.9 \%$ in the X-clamp model. Conversely, removing attendingfellow pair experience demonstrated a dramatic reduction in $R^{2}$ values from $7.2 \%$ to $2.7 \%$ for the CPB model and from $6.1 \%$ to $2.2 \%$ in the X-clamp model, suggesting that accounting for attending experience provides only a marginal improvement the patient risk-adjustment models only. Figure 3 graphically demonstrates the expected CPB and X-clamp times after adjusting for both patient risk and attending surgeon experience. As shown, there was a $44 \%$ and $36 \%$ reduction in CPB time and X-clamp time, respectively, over the duration of 50 collaborations.

TABLE 3. Multivariate crossclamp models

\begin{tabular}{lrrrrr}
\hline & \multicolumn{2}{c}{$\begin{array}{c}\text { Risk-adjustment } \\
\text { model }\end{array}$} & & \multicolumn{2}{c}{$\begin{array}{c}\text { Final cumulative } \\
\text { experience model }\end{array}$} \\
\cline { 2 - 3 } \multicolumn{1}{c}{ Term } & $\begin{array}{c}\text { Beta } \\
\text { estimate }\end{array}$ & Prob $>|\mathbf{t}|$ & & $\begin{array}{c}\text { Beta } \\
\text { estimate }\end{array}$ & Prob $>|\mathbf{t}|$ \\
\hline Intercept & 101.23 & $<.0001$ & & 142.48 & $<.0001$ \\
Age & -0.15 & .0008 & -0.17 & .0036 \\
Sex & -6.88 & $<.0001$ & -6.73 & $<.0001$ \\
Diabetes & -2.50 & .0096 & -3.91 & .0017 \\
Attending surgeon & & & -0.36 & $<.0001$ \\
$\quad$ experience & & & & & \\
Attending-fellow team & & & -0.89 & $<.0001$ \\
$\quad$ cumulative experience & & & & \\
\hline
\end{tabular}

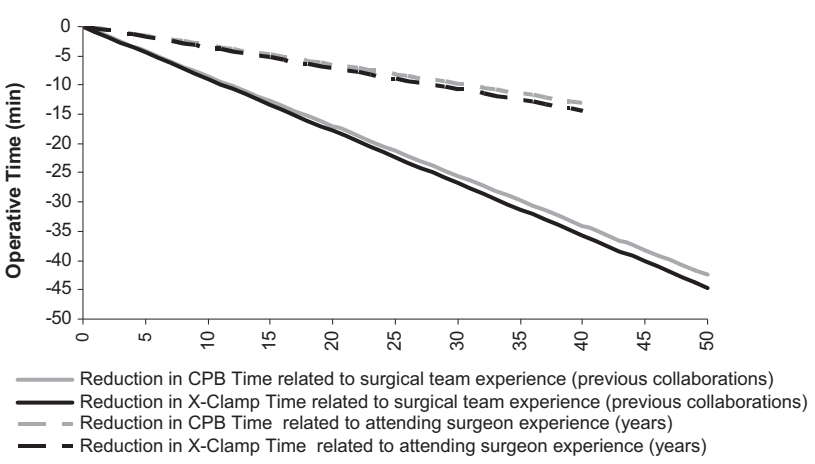

FIGURE 3. Relative influence of attending surgeon experience and cumulative experience on cardiopulmonary bypass $(C P B)$ and crossclamp (X-clamp) times.

\section{DISCUSSION}

Our analysis of more than 4000 isolated CABG procedures spanning nearly a decade and encompassing the clinical experience of 11 attending cardiac surgeons and 73 cardiothoracic fellows provides a number of important lessons. First, contrary to previous surgical learning curve studies, ${ }^{5}$ our study suggests that the attending surgeon learning curve may be clinically insignificant over the course of a cardiac surgeon's career performing $\mathrm{CABG}$ procedures when concomitantly integrating the cumulative experience of attending surgeon-fellow pairs. After adjusting for patient risk factors, each additional year of attending surgical experience reduced CPB and X-clamp times by 0.32 minutes and 0.36 minutes, respectively. Over the course of a 20 -year surgical career, this would translate into $<10 \%$ improvement. Second, we found the number of collaborations between an attending and his/her cardiothoracic fellow to be an extremely powerful variable in predicting operative efficiency (captured by the dependent variables of CPB time and X-clamp time). Our data suggested a dramatic and linear decline in both $\mathrm{CPB}$ and X-clamp times as the number of collaborations increased; by the time a team achieved 50 collaborations we detected a nearly $45 \%$ improvement in operative efficiency.

Our study generated findings that are consistent with the existing literature. First and foremost, these data reinforce the influence of cumulative team experience in achieving operative excellence. ${ }^{3,10,11}$ The primary etiology of improved surgical performance can be attributed to a number of factors, including accumulated learning by others that is effectively distributed among the group and improved teamwork and coordination. ${ }^{12}$ It is our contention that more effective teamwork is the most likely etiology, particularly in cardiac surgery where operating room teamwork deficiencies have been documented to have dire and life-threatening effects. ${ }^{13-15}$ Practically, more effective teamwork occurs as cardiothoracic fellows become more accustomed to a specific attending surgeon's technical and procedural preferences, learns the roles of other team members, and further clarifies his or her role as an 
assisting surgeon. ${ }^{12}$ The influence of cumulative experience on operative time has been well documented in other surgical subspecialties, including orthopedic surgery, which exhibited as much as a $10 \%$ reduction in operative time in teams with high levels of cumulative experience. ${ }^{12}$ Similar, but less significant results were also seen in laparoscopic cholecystectomy, where fixed teams achieved a lower procedure time. ${ }^{16}$ Finally, our research group recently found a similar effect in bilateral reduction mammoplasties; teams that performed 6 to 10 mammaplasties together had a nearly $15 \%$ reduction in operative time versus those that had minimal experience working together. ${ }^{17}$

Although our study is consistent with the existing literature on cumulative experience, the magnitude of the reduction was dramatic relative to other studies. With a $44 \%$ and $36 \%$ reduction in CPB and X-clamp times, respectively, the effect of cumulative experience far exceeds that seen in other surgical studies. A number of reasons may explain this finding. First, there is a general trend that low-technical complexity procedures generate less significant reductions in operative time compared with high-technical complexity procedures as a result of cumulative experience. ${ }^{12,16}$ Second, we were able to obtain high levels of cumulative experience due to a busy academic program with minimal attending surgeon turnover and a concentrated number of fellows. Whereas most studies are able to track cumulative experience to approximately 10 cases, our experience contains a substantial number of cumulative experiences that exceeds 50. By virtue of a significant number of patients in our study, a robust clinical database to allow for risk adjustment, and a broad array of cumulative experiences, we were able to generate statistically and clinically robust results that have not been demonstrated elsewhere.

Interestingly, we found that the attending surgical learning curve and the fellow surgical learning curve was clinically irrelevant (and statistically irrelevant for the latter). After patient risk adjustment, we found that $\mathrm{CPB}$ and X-clamp times decreased by fractions of a minute per year of surgeon experience. This finding differs markedly from existing surgeon learning curve studies, ${ }^{5,17}$ which have found both a clinical and statistical reduction in operative time as surgeon experience increases. It seems implausible that attending surgeons would gain marginal improvements in efficiency following fellowship graduation; however, there are unique features to cardiac surgery that may explain this finding. Compared with many other subspecialties such as plastic surgery, orthopedic surgery, and general surgery, cardiac surgery contains relatively few procedures (ie, CABG, valve replacements, and valve repairs), with cardiothoracic fellows beginning their training as proficient and highly competent general surgery graduates. This may result in attending surgeons achieving significant portion of their maturation as CABG surgeons during their cardiothoracic fellowship. There are some studies in the literature that reaffirm the absence of a clinically significant cardiac surgeon learning curve in procedures that are commonly taught during fellowship. ${ }^{18,19}$ Rather, learning curves in cardiac surgery tend to exist when new assistive technologies such as robotics, ${ }^{4,10,11}$ or new cardiac procedures such as transaortic/transapical aortic valves ${ }^{20}$ are introduced.

Our definition of operative efficiency and excellence is based on CPB and X-clamp times. There is little controversy that this period represents the high-risk period of a cardiac procedure. During CPB, the heart is stopped with perfusion to the brain and remainder of the body being delivered by nonpulsatile flow, which has well known and documented risks, such as stroke, renal failure, activation of the hemostatic system, and others. ${ }^{21} \mathrm{X}$-clamp time is the period following the initiation of the CPB where the aorta is X-clamped and the heart is rendered ischemic. In addition to the risks of $\mathrm{CPB}$, long X-clamp times can increase the risk of cardiovascular dysfunction following surgery. As a result, these measures are not only predictive of the team efficiency, but are directly related to patient complications and overall quality. Given the magnitude of the reductions in CPB and X-clamp times, these findings are clinically relevant with regard to patient outcomes. Unfortunately, due to a variable degree of missing complication data that resulted from the evolution of our database during the past decade, we chose not to evaluate complication data in this study.

\section{CONCLUSIONS}

We present the findings of a study that attempts to quantitatively assess both the learning curve of attending surgeon-cardiothoracic fellow pairs as well as the individual attending surgeon learning curve. We were able to identify that, after patient risk adjustment, the cumulative experience of an attending surgeon-cardiothoracic fellow combination has a dramatic effect on both CPB and X-clamp times - the high-risk segments of cardiac surgery procedures. Furthermore, and contrary to previous research, we found that attending surgeon learning curves following fellowship graduation are clinically insignificant. Taken together, these findings suggest that attending surgeons become highly proficient in CABG during their fellowship training and fail to make significant improvements over the course of their career. Furthermore, these findings indicate that the cumulative experience of an attending surgeon-cardiothoracic fellow team can have a significant influence on the efficiency of an operation (outcomes are improved by up to $50 \%$ when performed by high volume teams). These findings further reinforce the importance of effective teamwork and cumulative experience in cardiac surgery.

\section{Limitations}

This retrospective study spanning nearly a decade attempts to quantitatively analyze attending surgeon learning 
curves and the cumulative experience of attending surgeoncardiothoracic fellow teams. Although we utilized patient risk adjustment to optimize the accuracy of our findings there are some limitations that should be noted. This study was performed at an academic medical center where training is considered a major component of the mission. As such, it is unclear if these results are generalizable to other organizations that utilize a different patient care delivery model. Furthermore, the commitment to surgeon training may have influenced the results documented here and contributed to the clinically insignificant attending learning curve.

\section{References}

1. ElBardissi AW, Wiegmann DA, Dearani JA, Daly RC, Sundt TM 3rd. Application of the human factors analysis and classification system methodology to the cardiovascular surgery operating room. Ann Thorac Surg. 2007;83:1412-8; discussion 1418-9.

2. Wiegmann DA, ElBardissi AW, Dearani JA, Daly RC, Sundt TM 3rd. Disruptions in surgical flow and their relationship to surgical errors: an exploratory investigation. Surgery. 2007;142:658-65.

3. ElBardissi AW, Wiegmann DA, Henrickson S, Wadhera R, Sundt TM 3rd. Identifying methods to improve heart surgery: an operative approach and strategy for implementation on an organizational level. Eur J Cardiothorac Surg. 2008;34:1027-33.

4. Edmondson AC, Bohmer RM, Pisano GP. Disrupted routines: team learning and new technology implementation in hospitals. Admin Sci Q. 2001;46:685-716.

5. Carty MJ, Chan R, Huckman R, Snow D, Orgill DP. A detailed analysis of the reduction mammaplasty learning curve: a statistical process model for approaching surgical performance improvement. Plastic Reconstruct Surg. 2009;124:706-14.

6. List of variables for prediction of operative death for 1996 coronary artery bypass only procedures. The Cardiothoracic Surgery Network. Available from: http:// www.ctsnet.org/doc/4314.

7. Shahian DM, O'Brien SM, Filardo G, Ferraris VA, Haan CK, Rich JB, et al. The Society of Thoracic Surgeons 2008 cardiac surgery risk models: part 1-coronary artery bypass grafting surgery. Ann Thorac Surg. 2009;88(1 Suppl):S2-22.
8. Collett D. Modelling survival data in medical research. Boca Raton: CRC, Chapman Hall; 2003.

9. Ramsay CR, Grant AM, Wallace SA, Garthwaite PH, Monk AF, Russell IT. Statistical assessment of the learning curves of health technologies. Health Technol Assess. 2001;5:71-9.

10. Edmondson AC, Bohmer R, Pisano GP. Speeding up team learning. Harvard Business Rev. October 2001;79:125-32.

11. Pisano G. Organizational differences in rates of adoption of minimally invasive cardiac surgery. Mgmt Sci. 2001;46:752-68.

12. ElBardissi A. Individual experience and experience working together: predicting learning rates from knowing who knows what and knowing how to work together. Mgmt Sci. 2005;51:869-81

13. de Leval MR, Carthey J, Wright DJ, Farewell VT, Reason JT. Human factors and cardiac surgery: a multicenter study. J Thorac Cardiovasc Surg. 2000;119(4 Pt 1):661-72.

14. Catchpole KR, Giddings AE, Wilkinson M, Hirst G, Dale T, de Leval MR. Improving patient safety by identifying latent failures in successful operations. Surgery. 2007;142:102-10.

15. Carthey J, de Leval MR, Reason JT. Institutional resilience in healthcare systems. Qual Health Care. 2001;10:29-32.

16. Stepaniak PS, Vrijland WW, de Quelerij M, de Vries G, Heij C. Working with a fixed operating room team on consecutive similar cases and the effect on case duration and turnover time. Arch Surg. 2010;145:1165-70.

17. Xu R, Carty MJ, Orgill DP, Lipsitz SR, Duclos A. The teaming curve: a longitudinal study of the influence of surgical team familiarity on operative time. Ann Surg. In press.

18. Murzi M, Cerillo AG, Bevilacqua S, Gilmanov D, Farneti P, Glauber M. Traversing the learning curve in minimally invasive heart valve surgery: a cumulative analysis of an individual surgeon's experience with a right minithoracotomy approach for aortic valve replacement. Eur J Cardiothorac Surg. 2012;41:1242-6.

19. Song M-H, Tajima K, Watanabe T, Toshiaki I. Learning curve of coronary surgery by a cardiac surgeon in Japan with the use of cumulative sum analysis. Japan J Thorac Cardiovasc Surg. 2005;53:551-6.

20. Alli O, Booker J, Lennon R, Greason K, Rihal CS, Holmes DR Jr. Transcatheter aortic valve implantation: assessing the learning curve. JACC Cardiovasc Interv. 2012;5:72-9.

21. Sniecinski R, Chandler W. Activation of the hemostatic system during cardiopulmonary bypass. Anesth Analg. 2011;113:1319-33. 